\title{
Changing in the Reaction Time Causes the Confidence Matching in Group Decision Making
}

\author{
Jamal Esmaily', Reza Ebrahimpour ${ }^{1 *}$, Sajjad Zabbah² \\ ${ }^{1}$ Department of Computer Engineering, Shahid Rajaee Teacher Training University, Tehran, Iran \\ ${ }^{2}$ School of Cognitive Sciences, Institute for Research in Fundamental Sciences (IPM), Tehran, Iran
}

Article Info:

\section{ABSTRACT}

Introduction: Others' decision could constantly affect our decisions. There are numerous studies which revealed this effect properly. The social influence could affect most of the decision parameters, especially the confidence. Recently, an interesting study showed that participants tend to match their confidence to each other while they preserve their decision accuracy in a group decision making task. Since the decision accuracy is not able to explain this effect, the main question is which decision parameter is changing in a way which could be resulted in changing in the confidence and ultimately the confidence matching. Materials and Methods: In order to answer this question, ten subjects attended into a Psycho-Physic study with two separate sessions; Isolated and Social. In both situations, the participants were required to determine the direction of the presented motion dots and report their decision and confidence simultaneously. In the social situation, subjects were paired with four computergenerated partners, which had been created based on the data from the isolated session. The joint decisions were determined by the decision of either partner which had higher confidence. In this session, confidence and decision of the partner were represented to the participant. Results: The results indicated the subjects not only try to match their confidence to their partners, but also reported higher confidence in comparison with the isolated situation. We observed although the confidence matching did not affect the decision accuracy, however, the reaction time varied significantly in both sessions. Conclusion: This study reveals the role of reaction time in changing the confidence and consequently the confidence matching. This study once again emphasis on the reverse correlation of the reaction time and confidence even in a group decision making task.

\section{Key words:}

1. Reaction Time

2. Decision Making

3. Motion

*Corresponding Author: Reza Ebrahimpour

E-mail: rebrahimpour@srttu.edu 


\title{
تغيير زمان تصميمَيرى عامل انطباق قطعيت در تصميمات گروهى است
}

\author{
جمال اسماعيلى'، رضا ابراهيم يور'": سجاد ذباح' \\ 'دانشكده مهندسى كامييوتر، دانشكاه تربيت دبير شهيد رجايى، تهران، ايران \\ 'يَّروهشكده علوم شناختى، يُروهشكاه دانشهاى بنيادى، تهران، ايران
}

كليد وازهها:

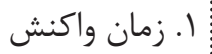

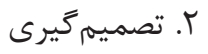

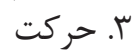

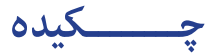

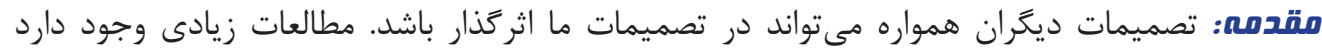

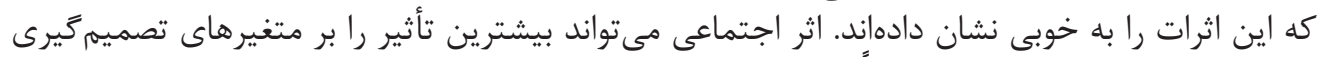

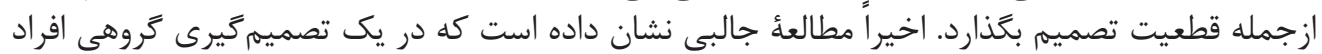

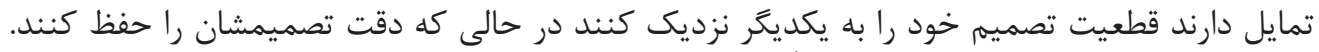

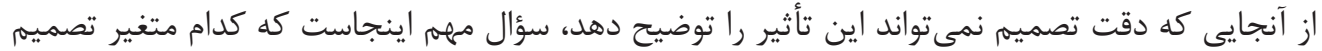

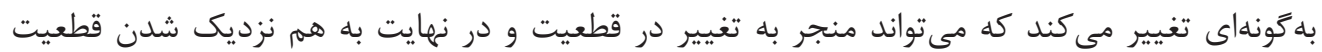

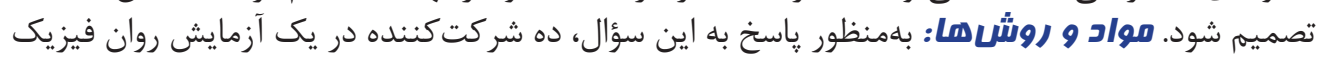

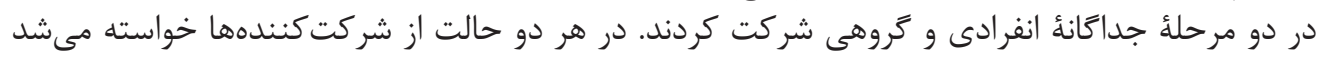

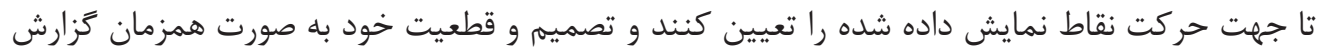

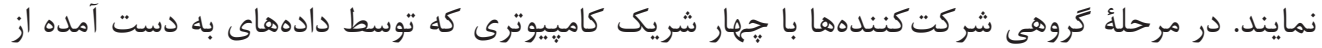

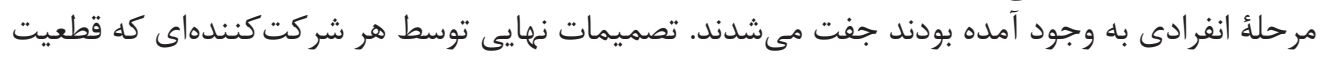

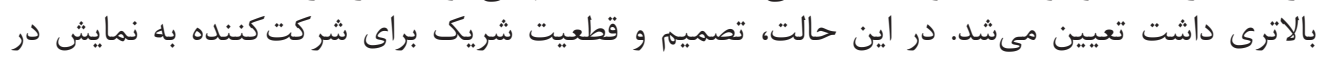

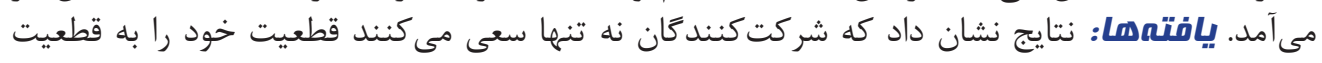

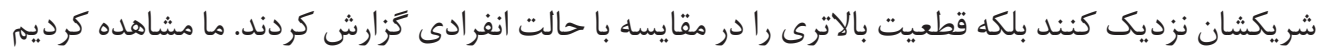

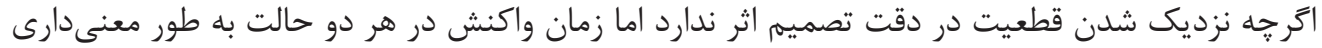

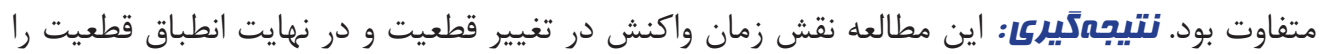

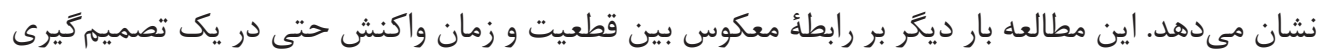
كروهى تأكيد دارد.

" نويسنده مسئول: رضا ابراهيم يور آدرس الكترونيكى: rebrahimpour@srttu.edu 
مشـخص كنـد تصويسـر نمايسـ داده شــده در سـمت جــ

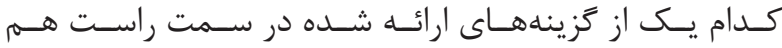

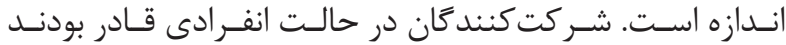

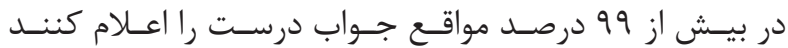

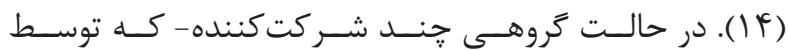

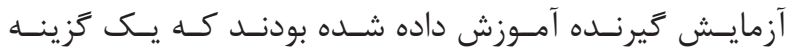

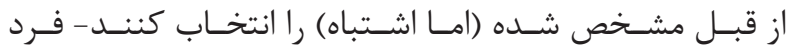

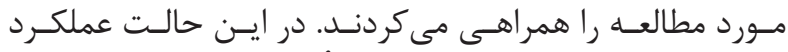

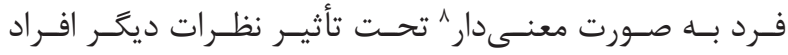

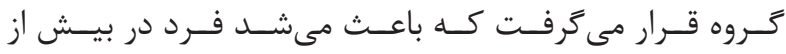

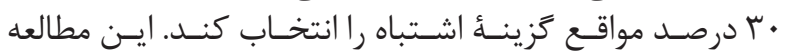

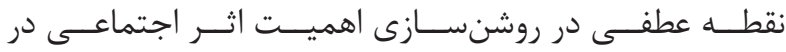

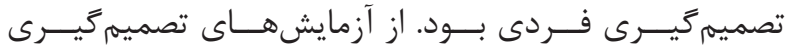

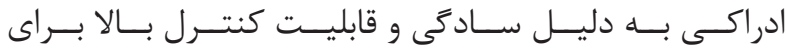

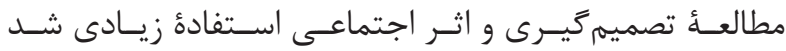

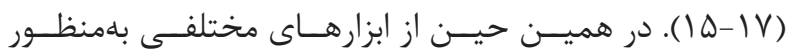

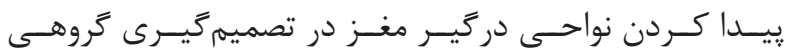

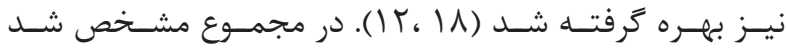

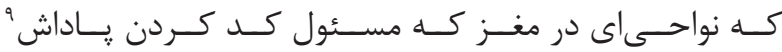

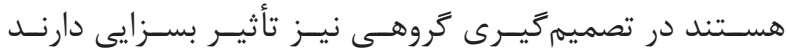

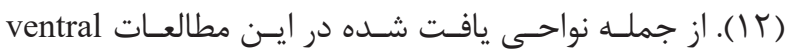
striatum, caudate nucleus, orbitofrontal cortex, insula, anterior cingulate cortex

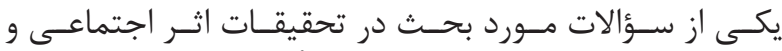

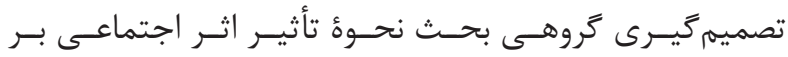

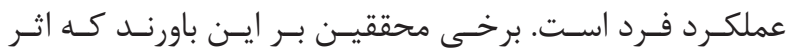

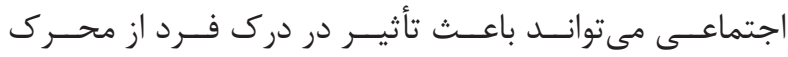

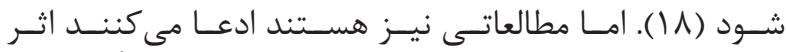

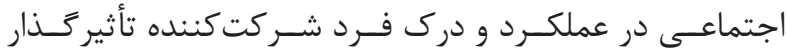

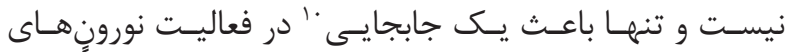

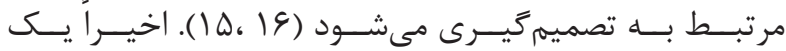

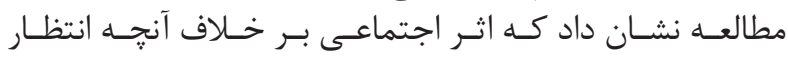

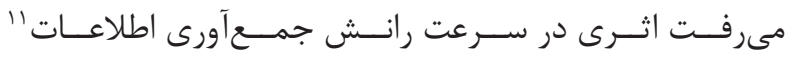

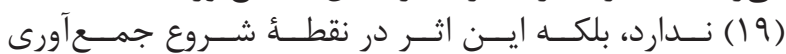

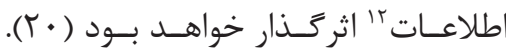

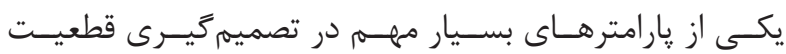

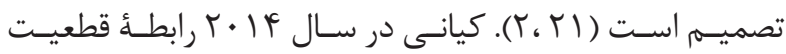

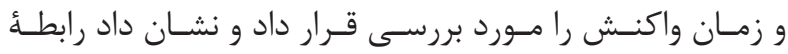

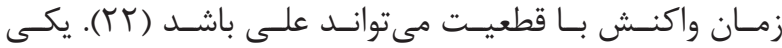

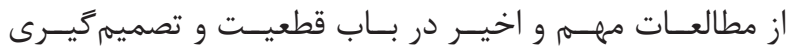

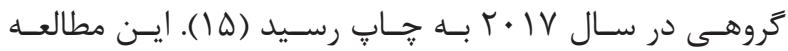

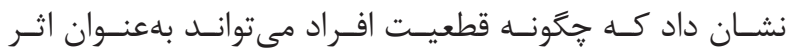

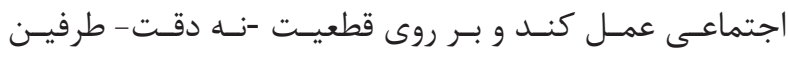

${ }^{1}$ Evidence accumulation

${ }^{2}$ Perceptual

${ }^{3}$ Random dot motion

${ }^{4}$ Multi-stage

${ }^{5}$ Multiple choice

${ }^{6}$ Discrete decision making

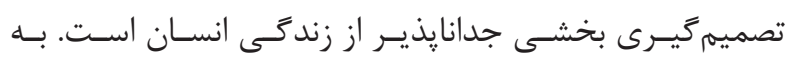

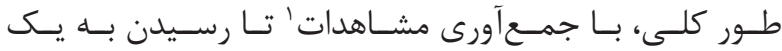

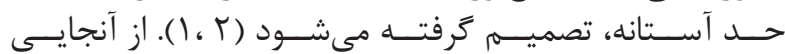

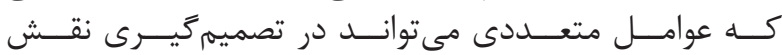

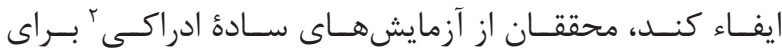

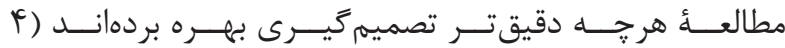

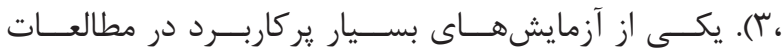

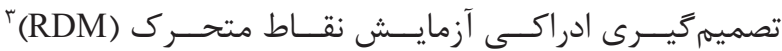

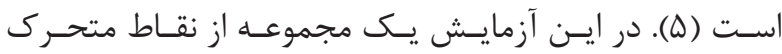

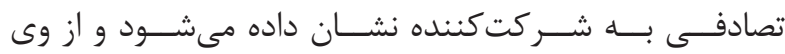

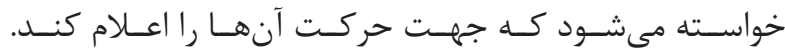

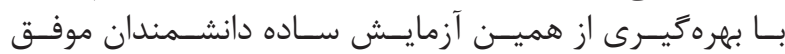

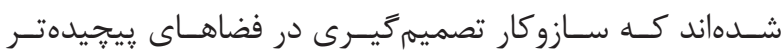

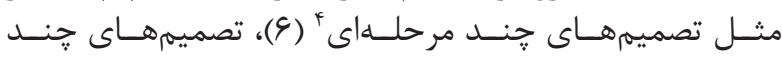

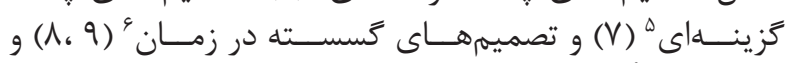

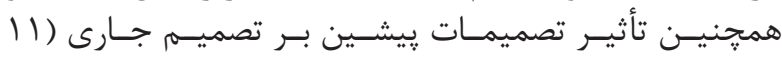

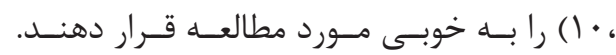

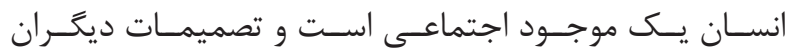

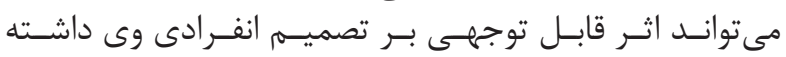

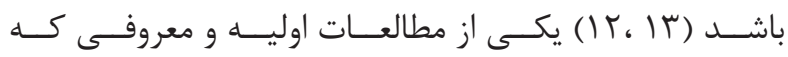

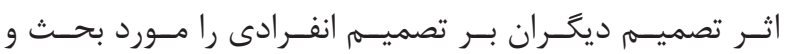

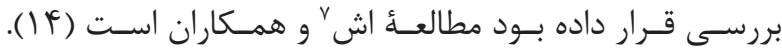

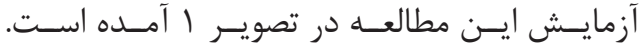
در ايـن آزمايسش از فـرد مـورد مطالعـهـ خواسـته مىشـود كـهـ

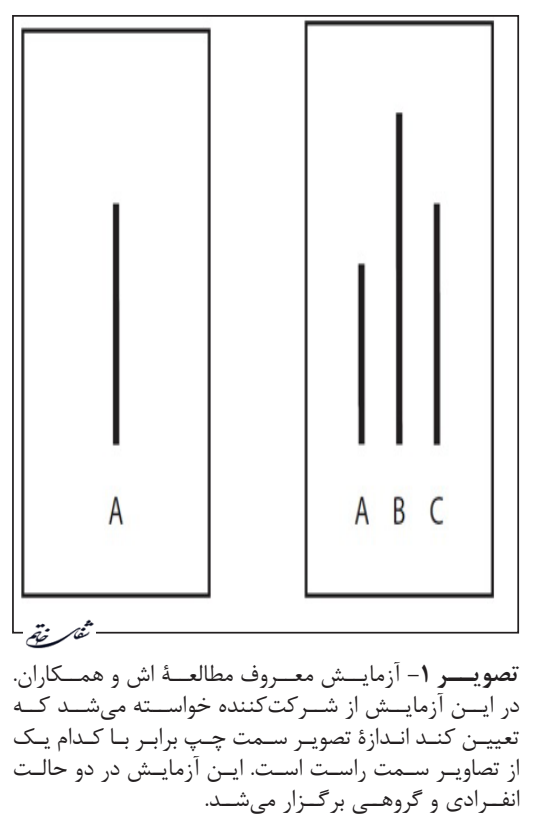

${ }^{7}$ Acsh

${ }^{8}$ Significant

${ }^{9}$ Reward coding

${ }^{10}$ Shift

${ }^{11}$ Drift rate

${ }^{12}$ Starting point 


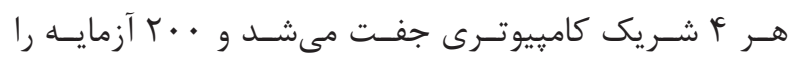

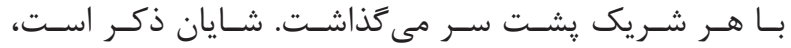

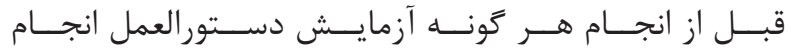

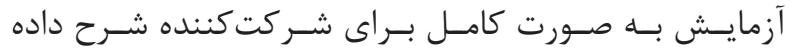

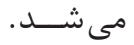

\section{جعَونكَى انجام آزمايش}

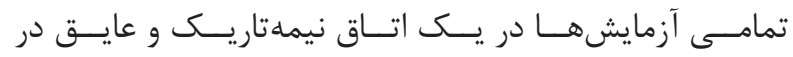

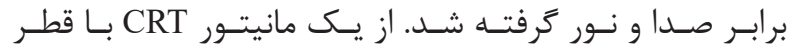

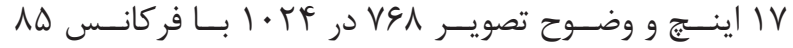

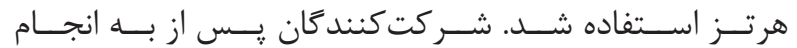

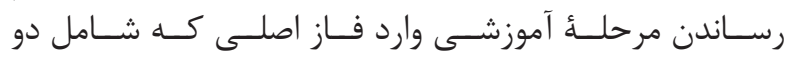

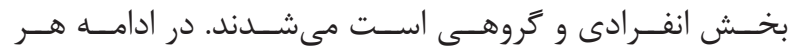

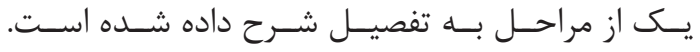

مرحلة انفرادى

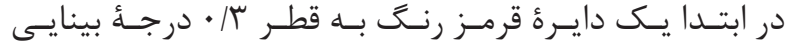

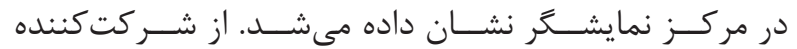

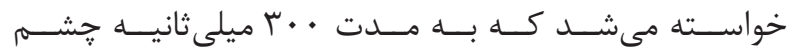

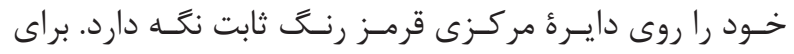

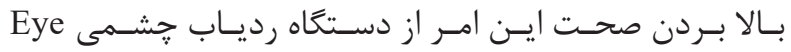
Link 1000 SR Research

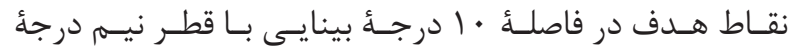

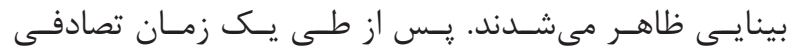

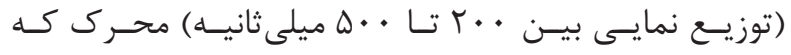

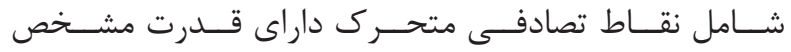

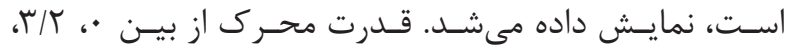

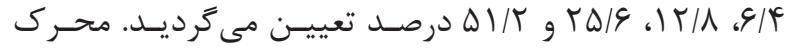

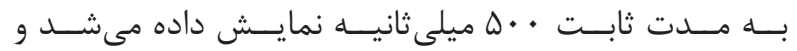

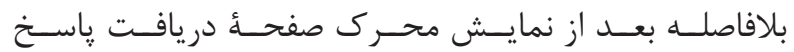

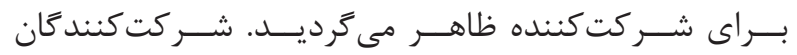

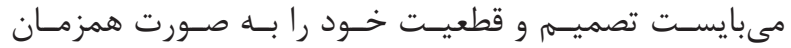

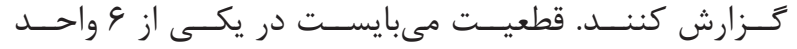

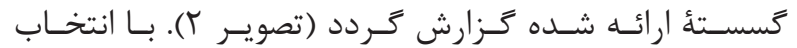

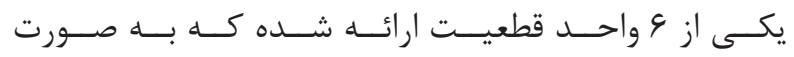

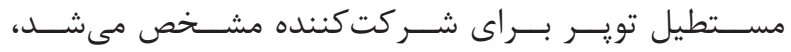

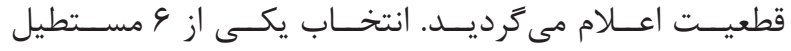

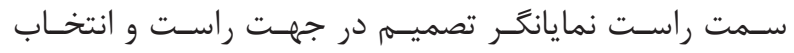

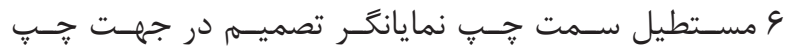

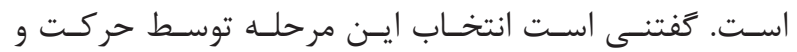

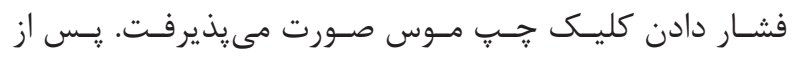

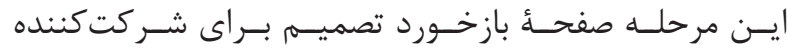

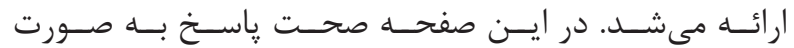

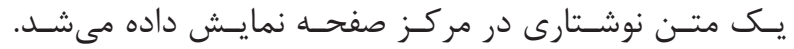

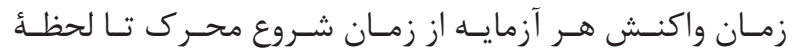

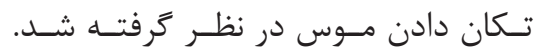

${ }^{13}$ Psychophysics

${ }^{14}$ Psychometric function

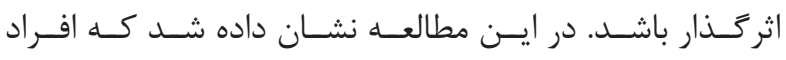

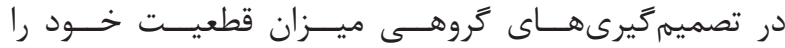

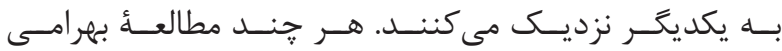

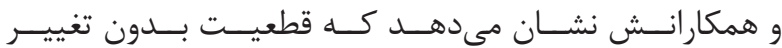

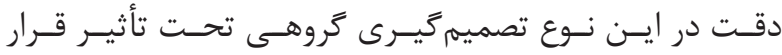

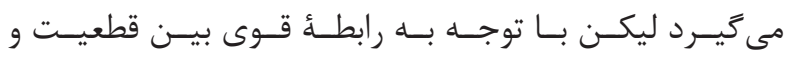

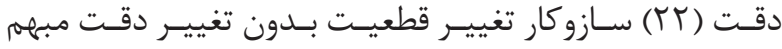

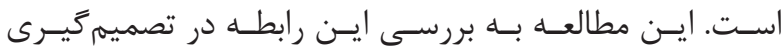

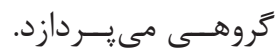

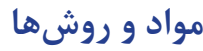

روش تحقيق

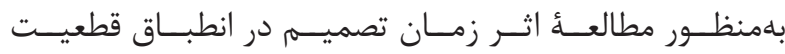

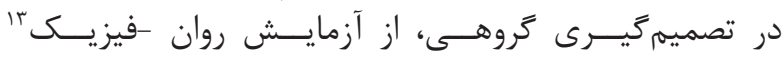

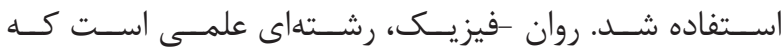

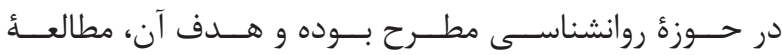

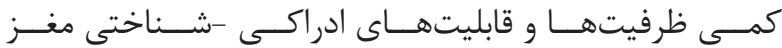

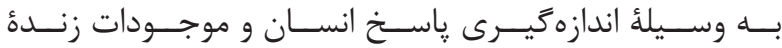

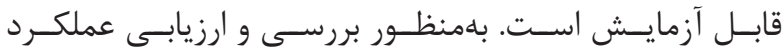

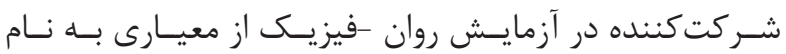

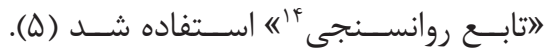
جمع آورى داده

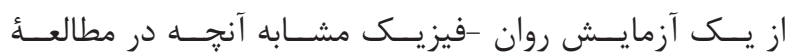

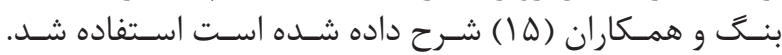

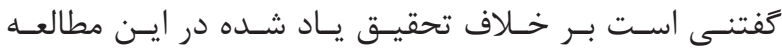

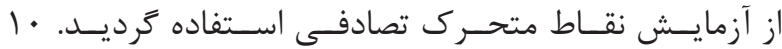

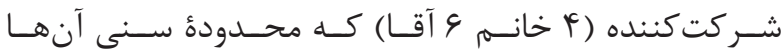

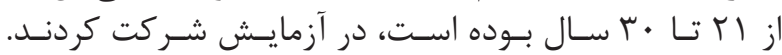

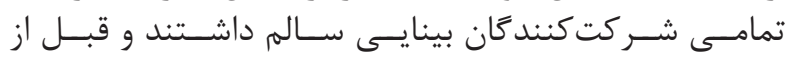

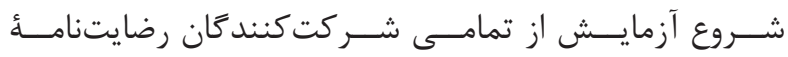

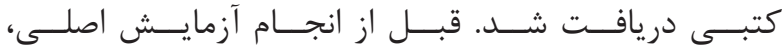

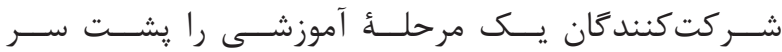

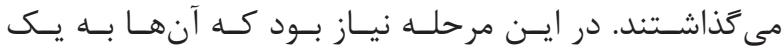

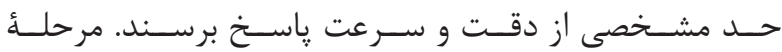

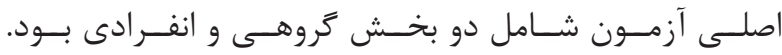

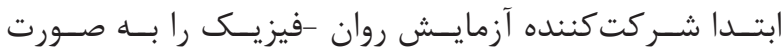

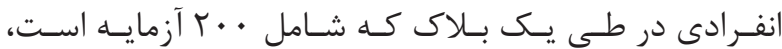

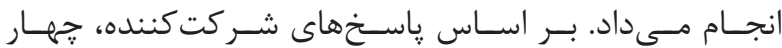

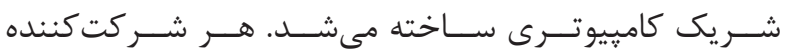

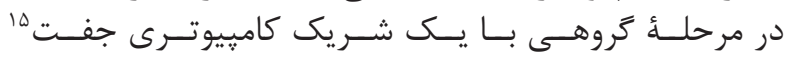

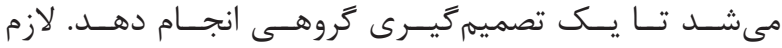

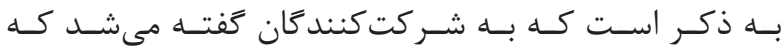

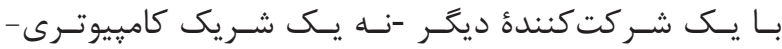

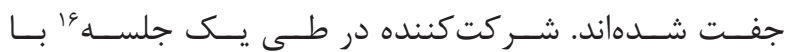




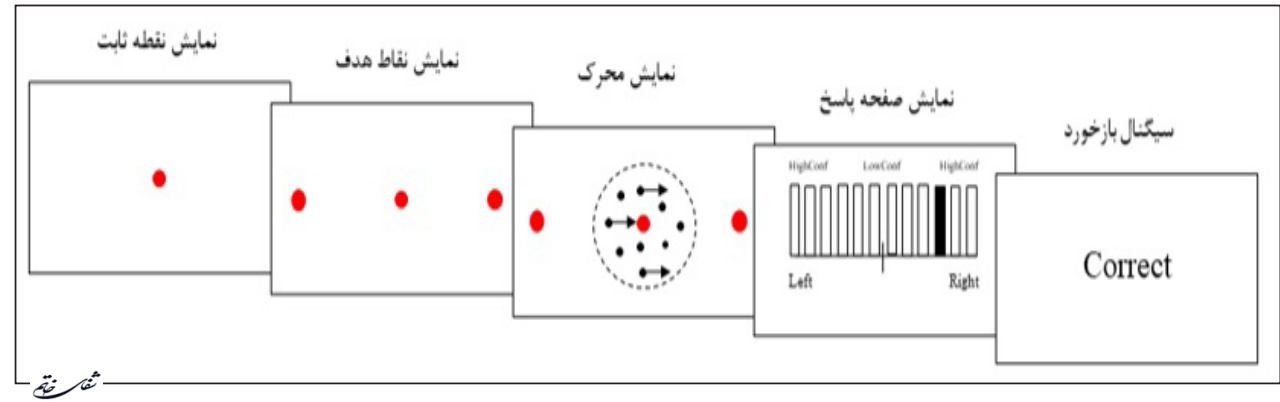

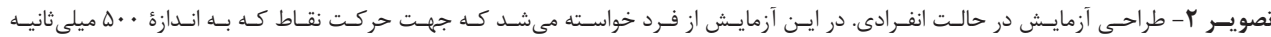

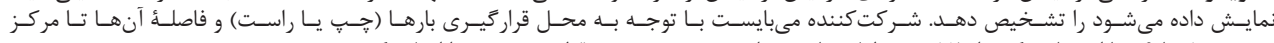

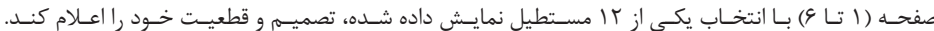

\section{محرك بينايى}

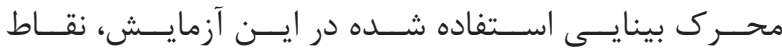

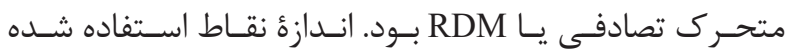

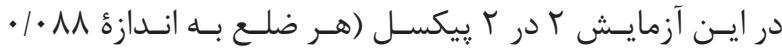

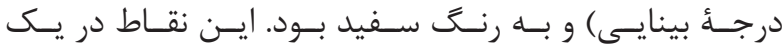

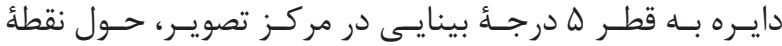

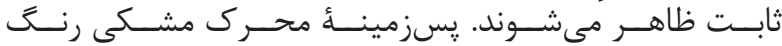

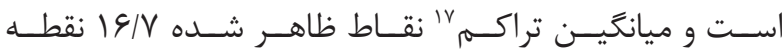

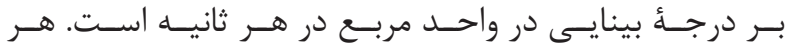

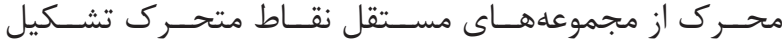

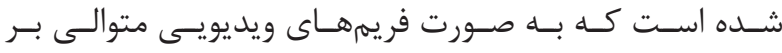

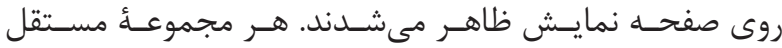

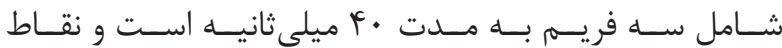

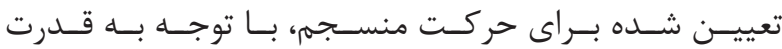

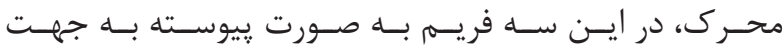

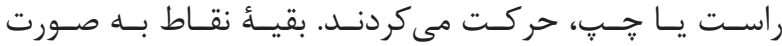

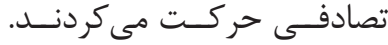

$$
\begin{aligned}
& \text { شريك كاميبيوترى }
\end{aligned}
$$

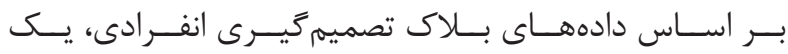

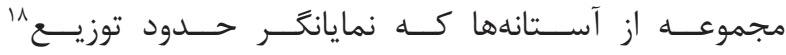

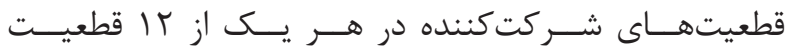

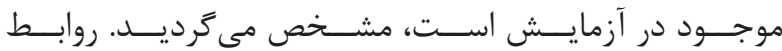

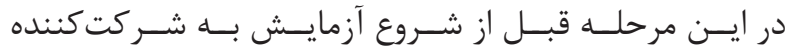

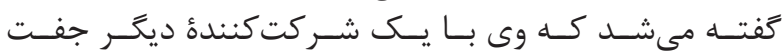

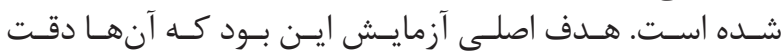

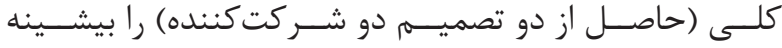

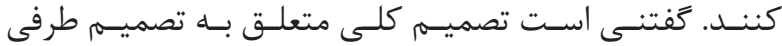

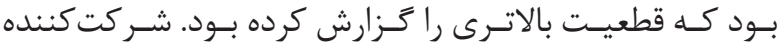

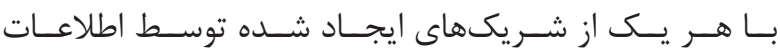

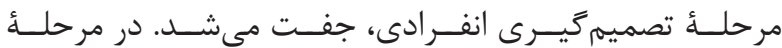

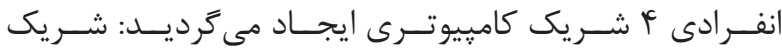

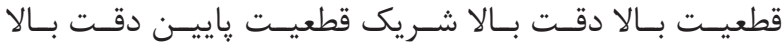

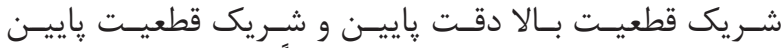

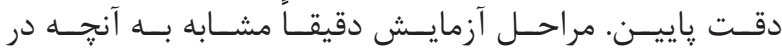

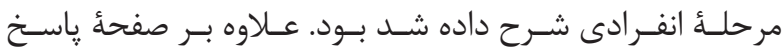

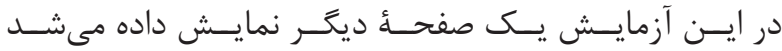

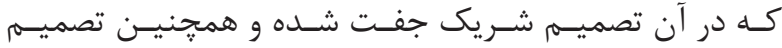

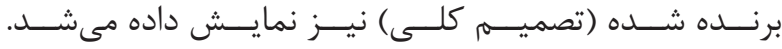

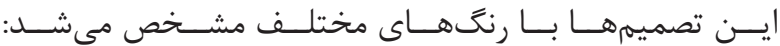

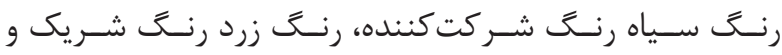

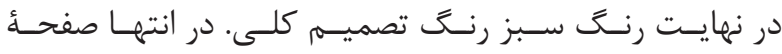

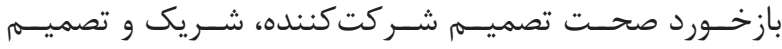

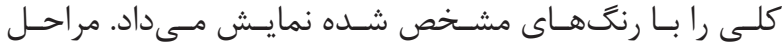

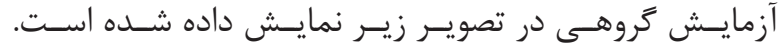

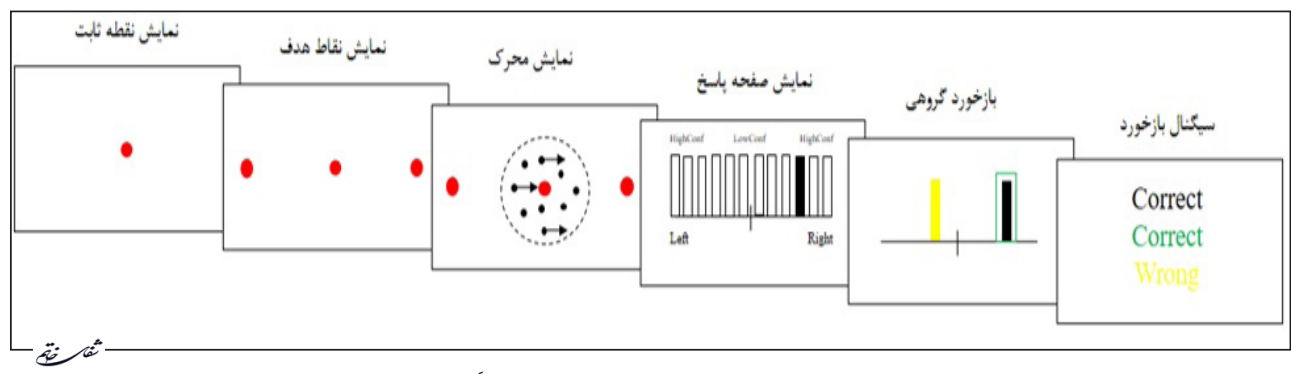

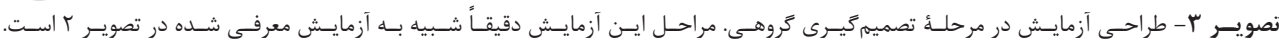

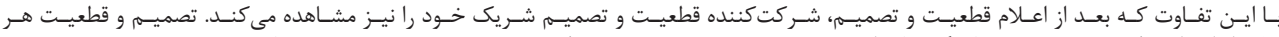

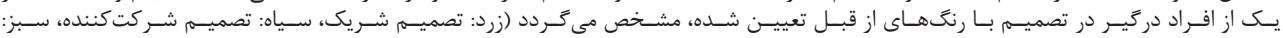

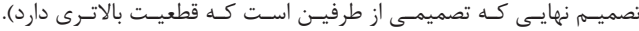

${ }^{17}$ Density

${ }^{18}$ Distribution

${ }^{19}$ Normal cumulative distribution 


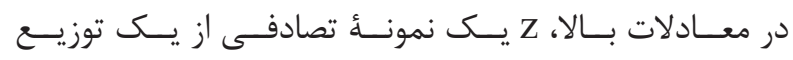

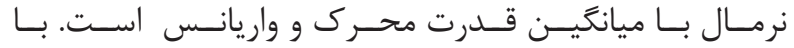

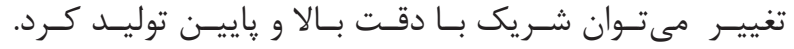

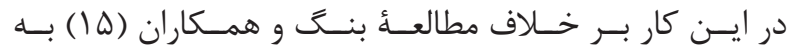

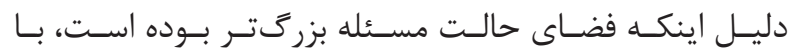

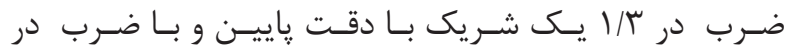

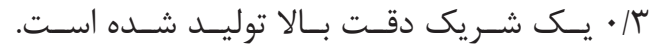

$$
\text { تحليل آمارى دادهها }
$$

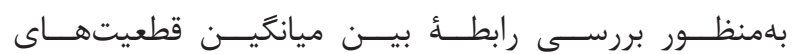

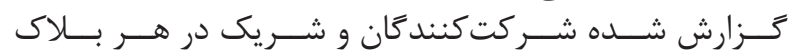

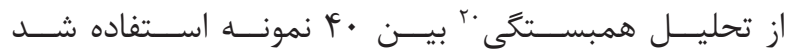

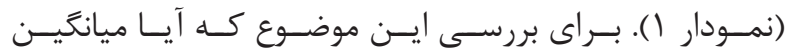

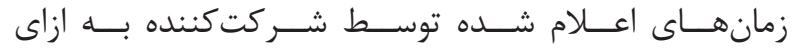

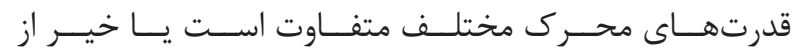

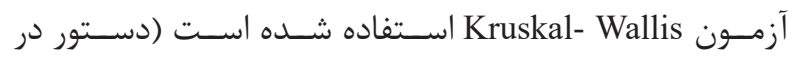

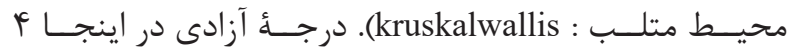

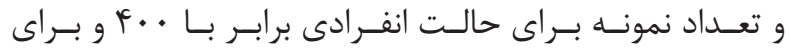

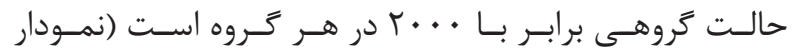

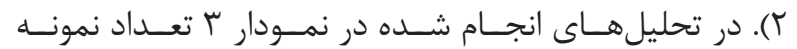

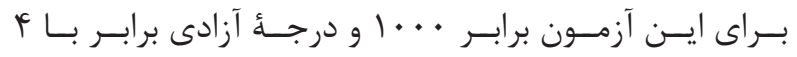

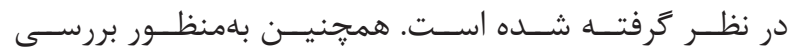

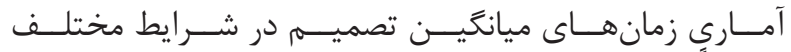

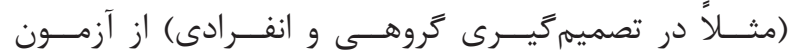

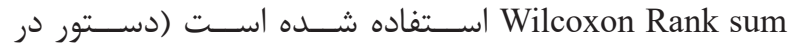

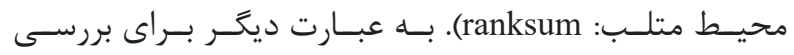

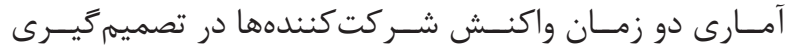

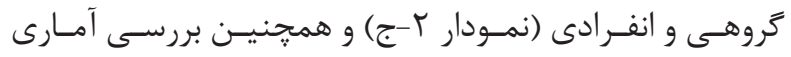

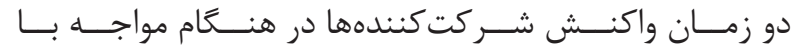

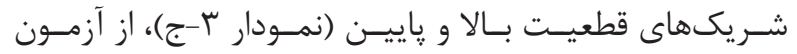

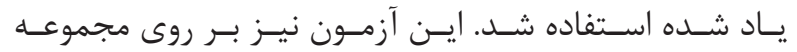

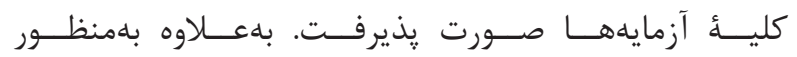

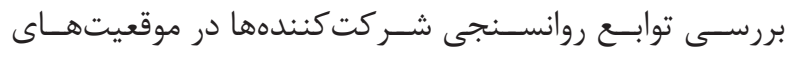

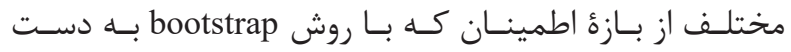

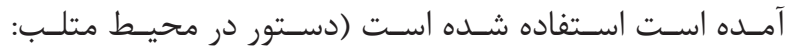
bootci

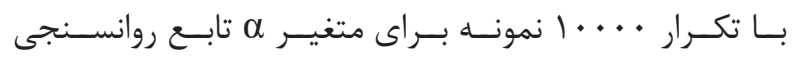

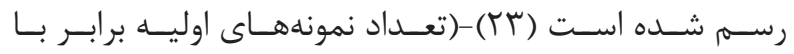

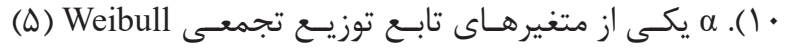

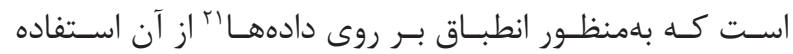

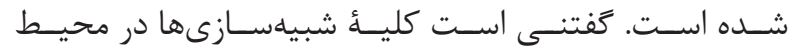

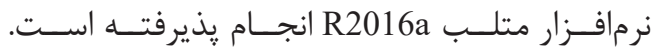

بافتهها

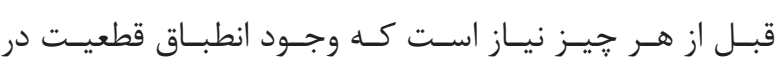

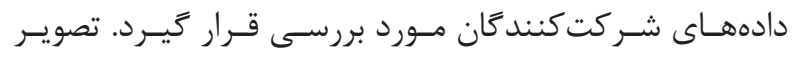

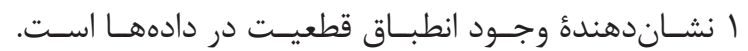

مربـوط بـهـ يافتـن ايـن حـدود آسـتانه در ادامـه آمـده اسـت:

$$
\begin{gathered}
\sum_{j \leq i} p j=\frac{1}{10} \sum_{s \epsilon S} \Phi\left(\frac{\theta i-s}{\sigma}\right) \\
p(r=i \mid s)=\left\{\begin{array}{cc}
\Phi\left(\frac{\theta_{-6}-s}{\sigma}\right) & i=-6 \\
\Phi\left(\frac{\theta_{-i}-s}{\sigma}\right)-\Phi\left(\frac{\theta_{i-1}-s}{\sigma}\right) & -6<i<6 \\
1-\Phi\left(\frac{\theta_{5}-S}{\sigma}\right) & i=6
\end{array}\right.
\end{gathered}
$$

$$
a_{\text {agent }}=\frac{\sum_{s \in S, s>0} \sum_{i=1}^{6} p_{i, s}+\sum_{s \in S, s<0} \sum_{i=-6}^{-1} p_{i, s}}{10}
$$

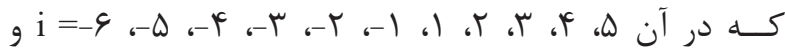

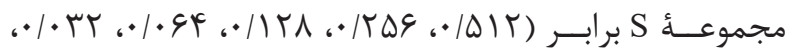
م هi

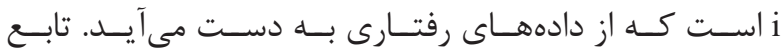

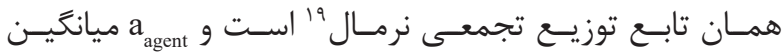

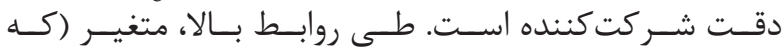

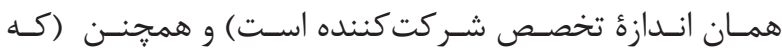

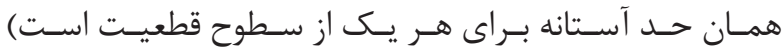

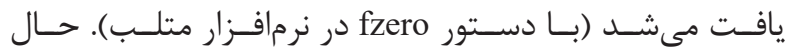

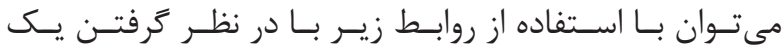

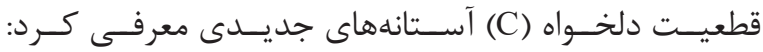

$$
\mathrm{p}_{\mathrm{i}}=\frac{e^{i \lambda}}{\sum_{j=1}^{6} e^{i \lambda}}
$$

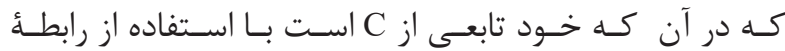

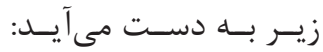

$$
c=\frac{\sum_{j=1}^{6} j e^{j \lambda}}{\sum_{j=1}^{6} e^{j \lambda}}
$$

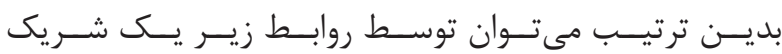

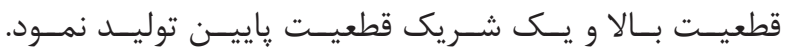

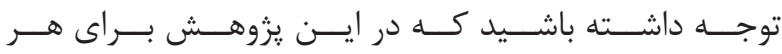

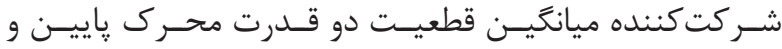

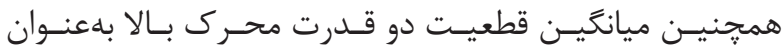

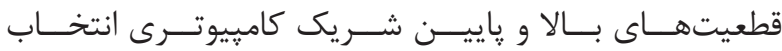

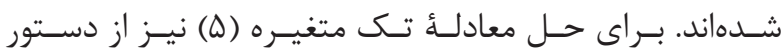

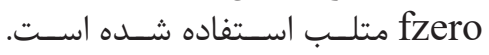

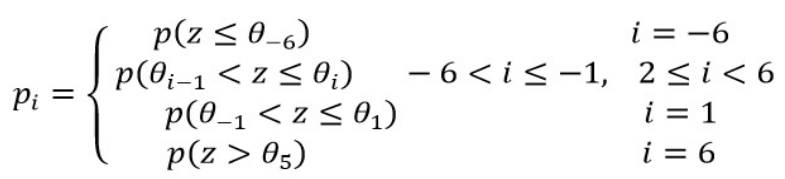




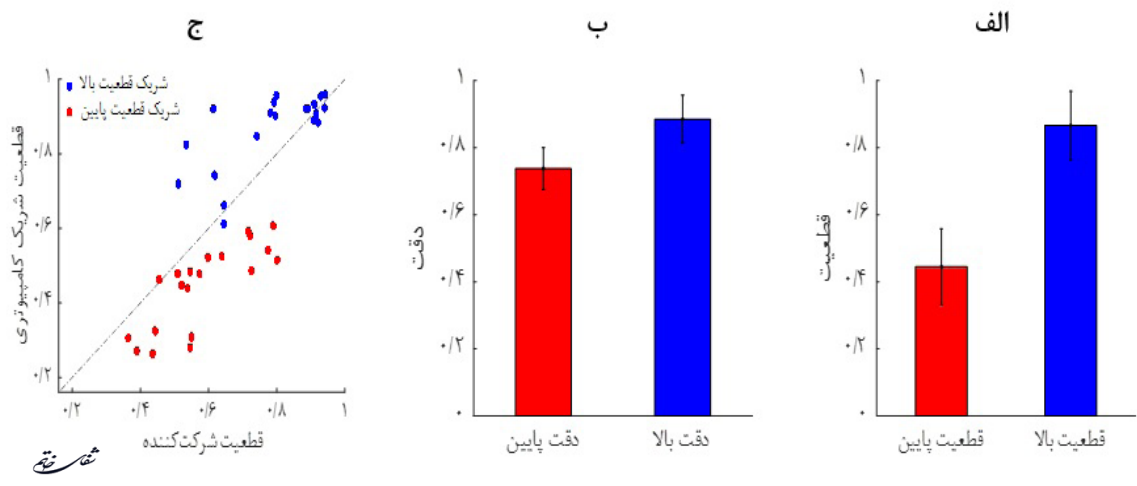

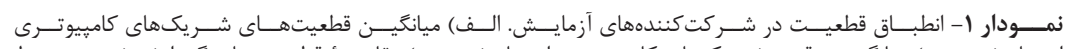

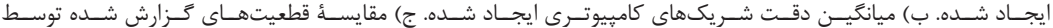

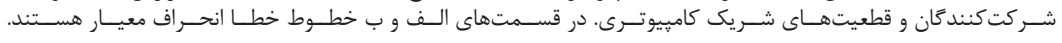

ج

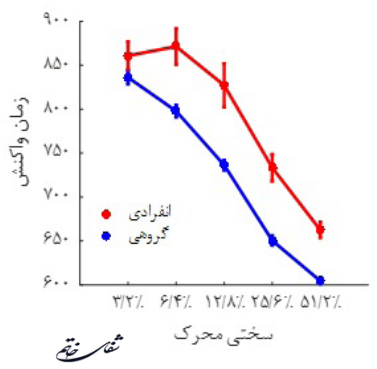

ب

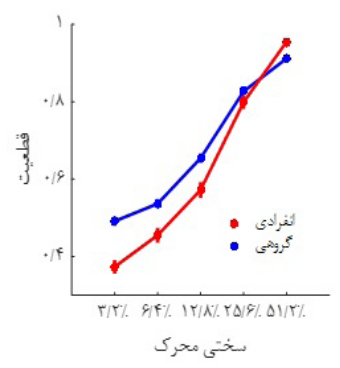

الف

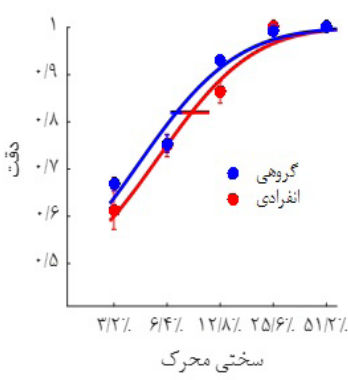

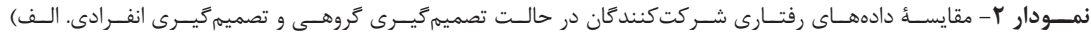

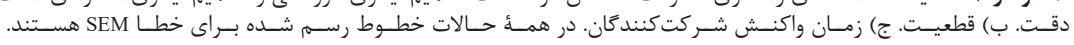

ج

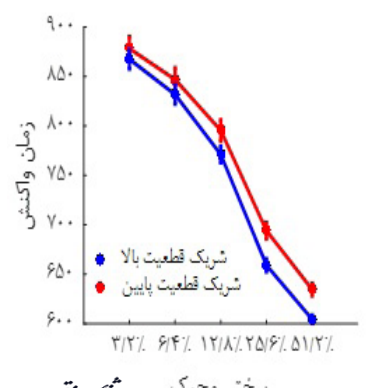

ب

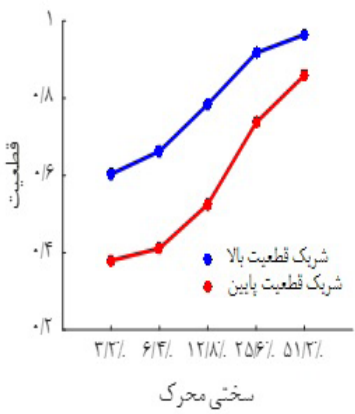

الف

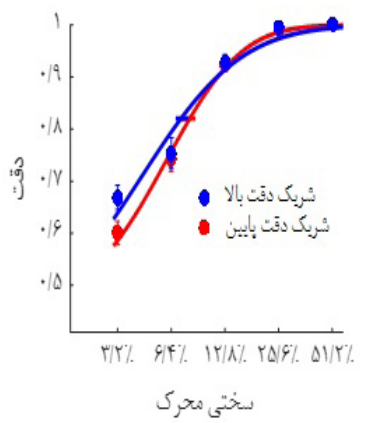

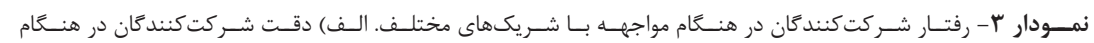

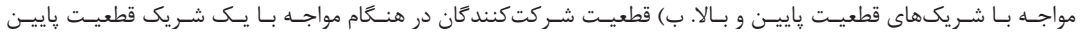

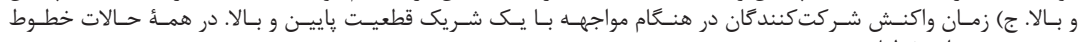

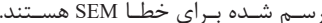

$$
\text { خـــود را تــزارش مى دادنــد. }
$$

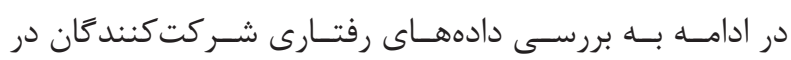

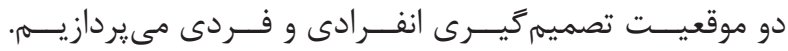

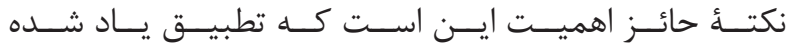

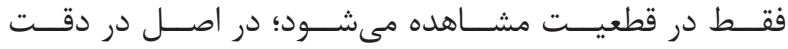

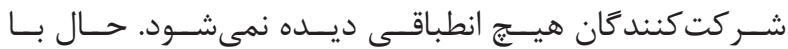

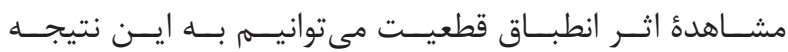

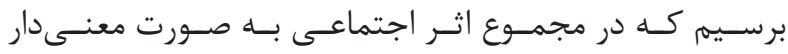

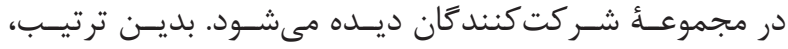

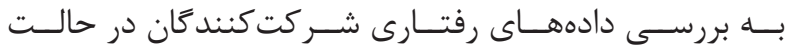

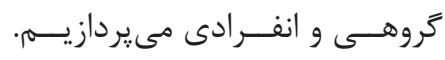

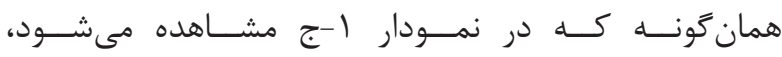

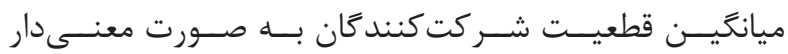

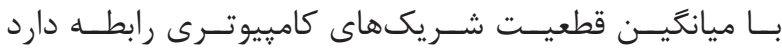

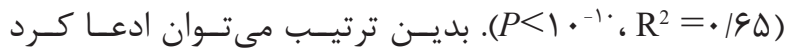

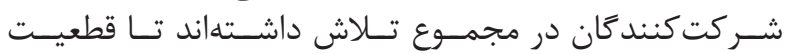

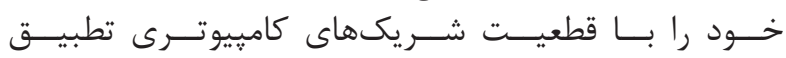

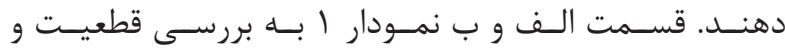

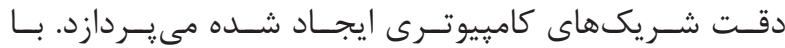

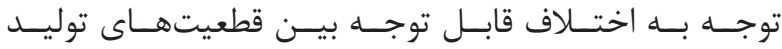

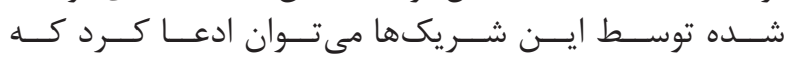

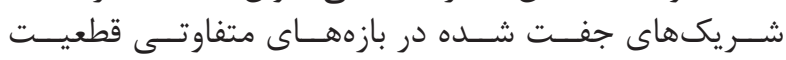




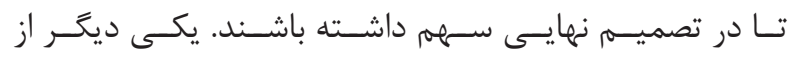

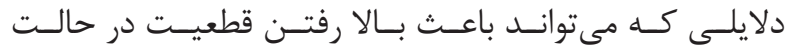

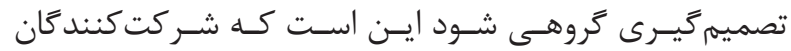

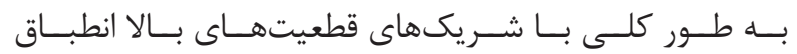

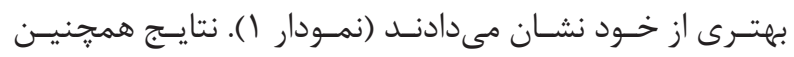

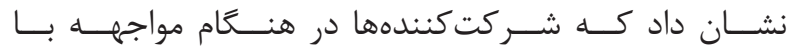

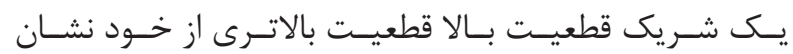

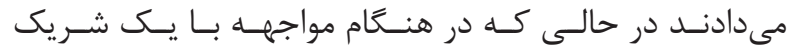

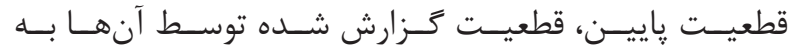

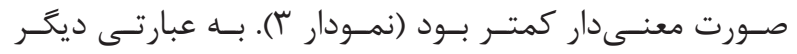

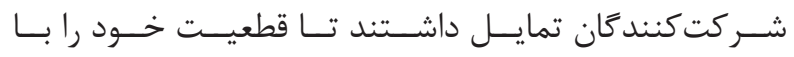

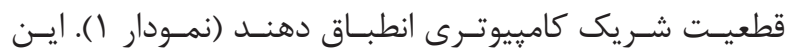

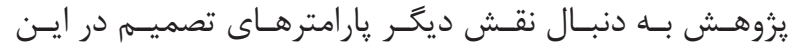

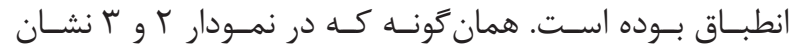

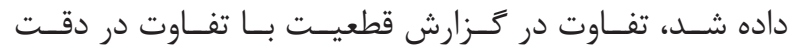

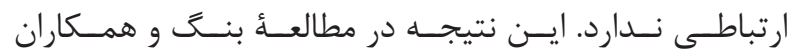

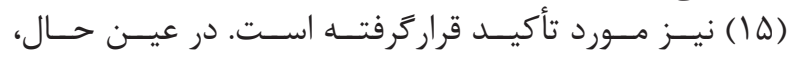

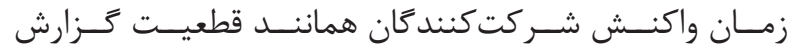

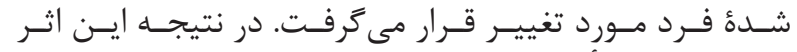

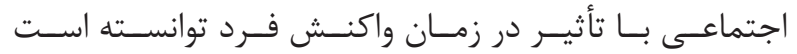

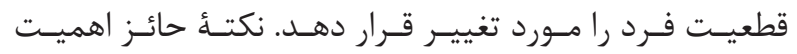

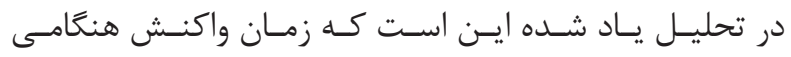

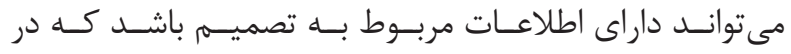

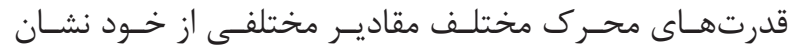

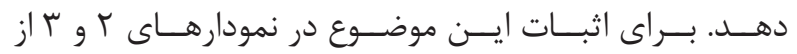

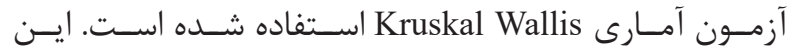

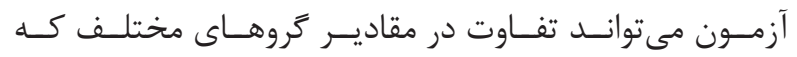

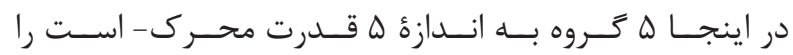

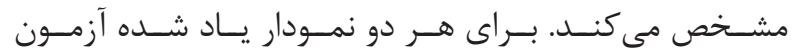

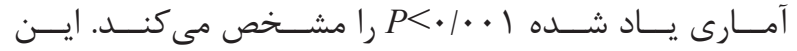

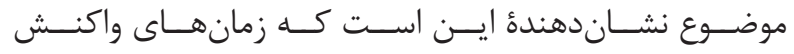

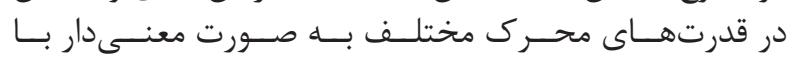

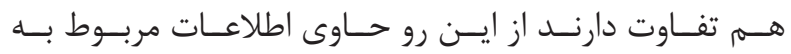

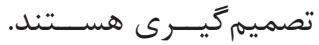

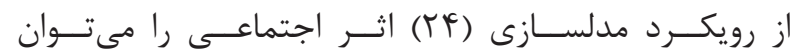

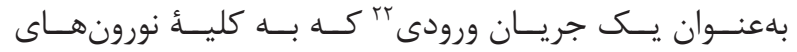

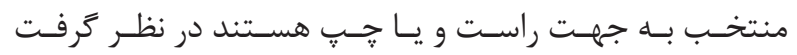

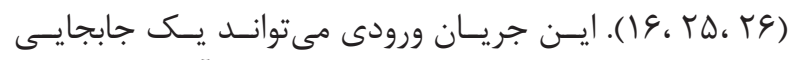

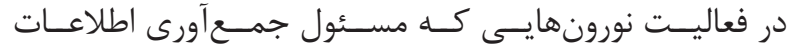

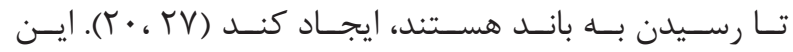

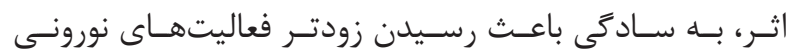

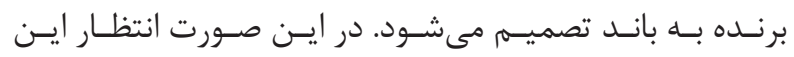

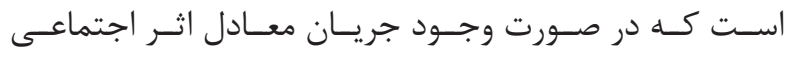

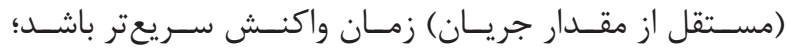

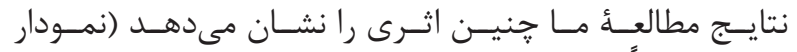

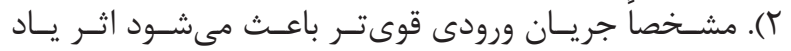

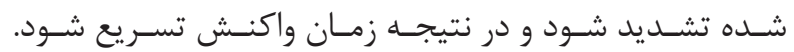

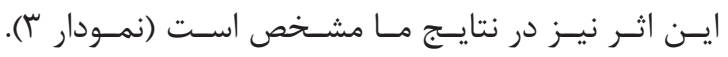

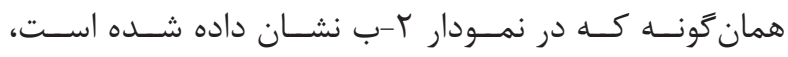

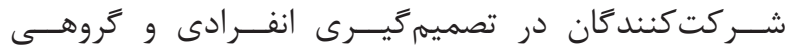

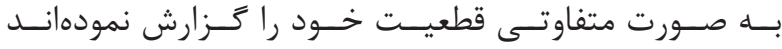

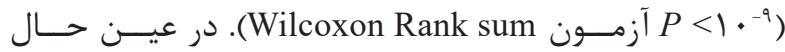

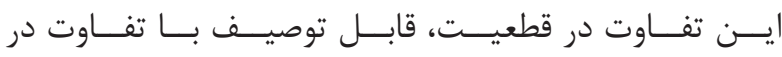

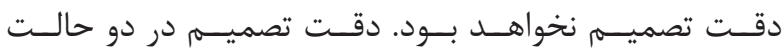

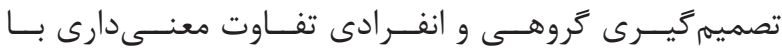

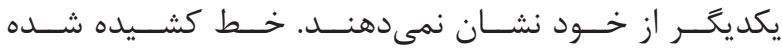

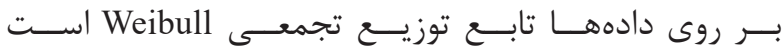

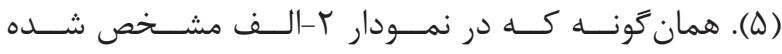

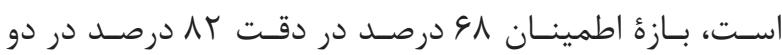

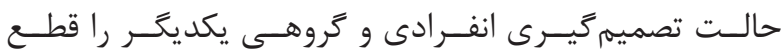

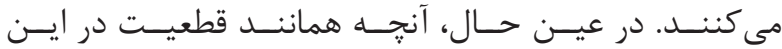

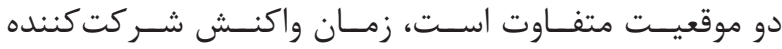

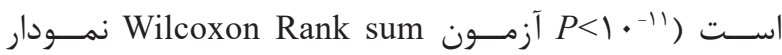

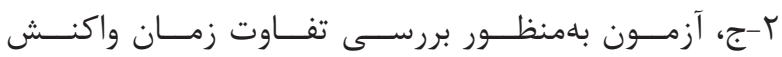

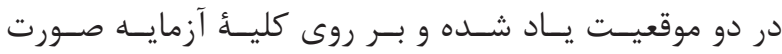

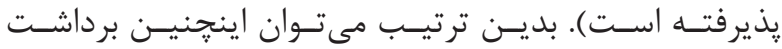

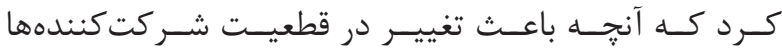

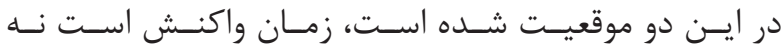

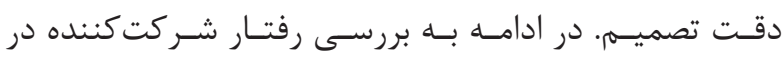

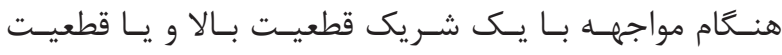

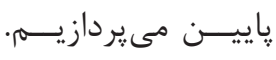

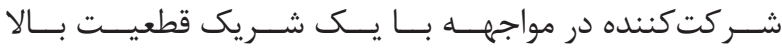

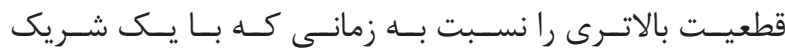

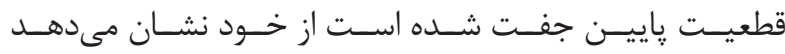

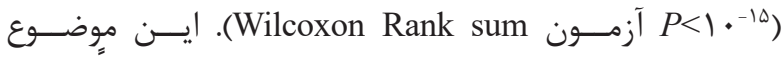

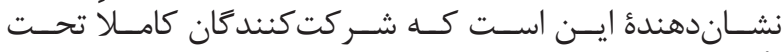

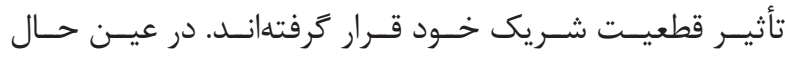

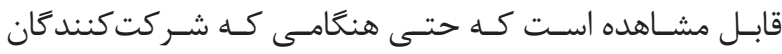

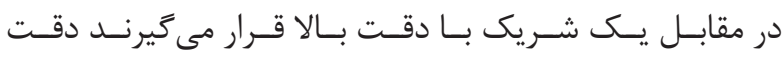

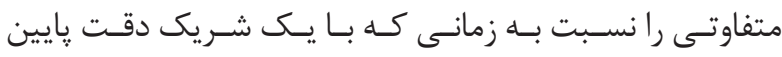

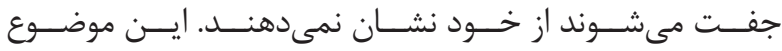

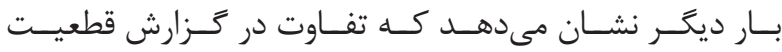

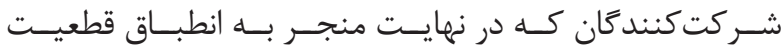

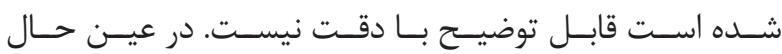

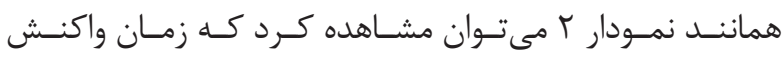

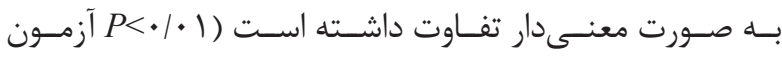

.(Wilcoxon Rank sum

بحث و نتيجه كيرى

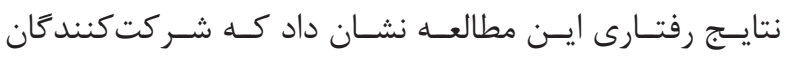

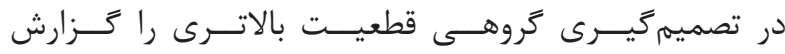

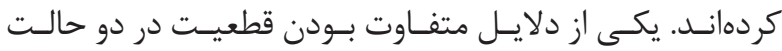

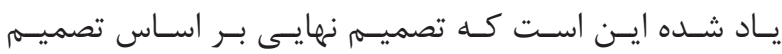

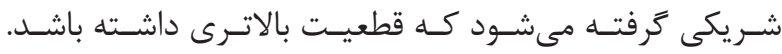

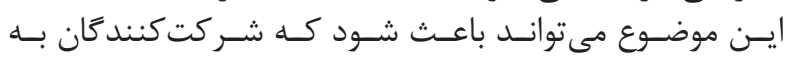

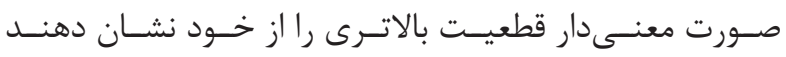




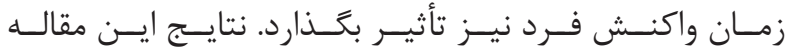

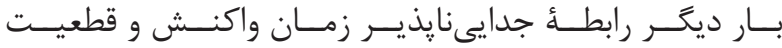

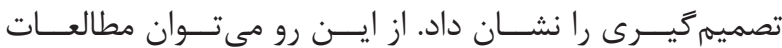

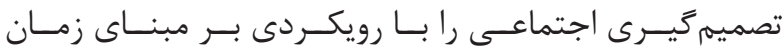

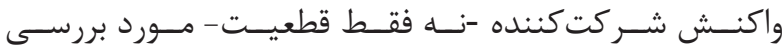

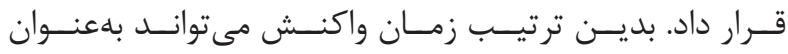

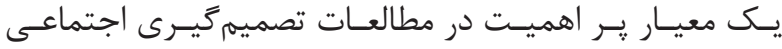

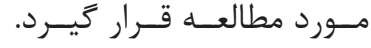

$$
\begin{aligned}
& \text { تشكر و قلر دانى } \\
& \text { ايسن تحقيـق بــا حمايـت مالـى دانشـعاه تربيـت دبيـر شـــيد }
\end{aligned}
$$

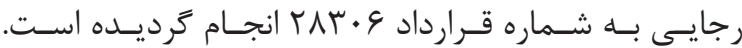

1. Hanks TD, Summerfield C. Perceptual decision making in rodents, monkeys, and humans. Neuron. 2017; 93(1): 15-31.

2. Gold JI, Shadlen MN. The neural basis of decision making. Annu Rev Neurosci. 2007; 30(1): 535-74.

3. Britten KH, Shadlen MN, Newsome WT, Movshon JA. The analysis of visual motion: a comparison of neuronal and psychophysical performance. J Neurosci. 1992; 12(12): 4745-65.

4. Shadlen MN, Newsome WT. Neural basis of a perceptual decision in the parietal cortex (area lip) of the rhesus monkey. J Neurophysiol. 2001; 86(4): 1916-36.

5. Roitman JD, Shadlen MN. Response of neurons in the lateral intraparietal area during a combined visual discrimination reaction time task. J Neurosci. 2002; 22(21): 9475-89.

6. van den Berg R, Zylberberg A, Kiani R, Shadlen MN, Wolpert DM. Confidence is the bridge between multistage decisions. Curr Biol. 2016; 26(23): 3157-68.

7. Churchland AK, Kiani R, Shadlen MN. Decisionmaking with multiple alternatives. Nat Neurosci. 2008; 11(6): 693-702.

8. Kiani R, Churchland AK, Shadlen MN. Integration of direction cues is invariant to the temporal gap between them. The Journal of Neuroscience. 2013; 33(42): 16483-9.

9. Zabbah S. The role of the primary information on importance of the last information in decision making. Shefaye Khatam. 2016; 4(4): 26-34.

10. Olianezhad F. The influence of past decision information on decision making in the present. Shefaye

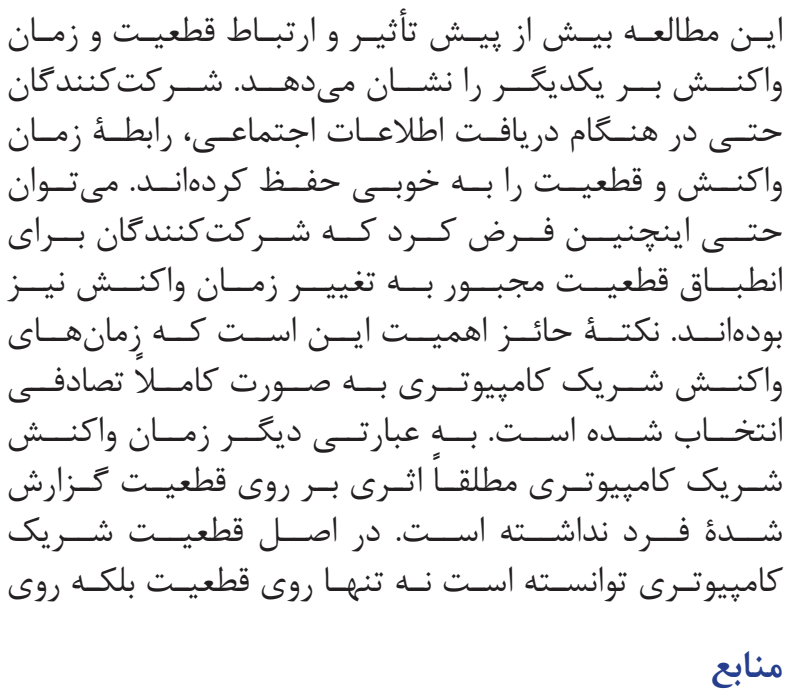

Khatam. 2016; 4(3): 1-8.

11. Purcell BA, Kiani R. Neural mechanisms of post-error adjustments of decision policy in parietal cortex. Neuron. 2016; 89(3): 658-71.

12. Mojzisch A, Krug K. Cells, circuits, and choices: social influences on perceptual decision making. C Cogn Affect Behav Neurosci. 2008; 8(4): 498-508.

13. Ruff CC, Fehr E. The neurobiology of rewards and values in social decision making. Nat Rev Neurosci. 2014; 15(8): 549-62.

14. Asch SE. Studies of independence and conformity: I. A minority of one against a unanimous majority. Psychological Monographs. 1956; 70(9): 1-70.

15. Bang D, Aitchison L, Moran R, Herce Castanon S, Rafiee B, Mahmoodi A, et al. Confidence matching in group decision-making. Nature Human Behaviour. 2017; 1(6): 1-17.

16. Germar M, Schlemmer A, Krug K, Voss A, Mojzisch A. Social influence and perceptual decision making. Pers Soc Psychol Bull. 2014; 40(2): 217-31.

17. Kelvin P, Deschamps J-C. Social influence and social change. Serge Moscovici, Academic Press, (European Monographs in Social Psychology), London, 1976. European Journal of Social Psychology. 1979; 9(4): 441-6.

18. Berns GS, Chappelow J, Zink CF, Pagnoni G, MartinSkurski ME, Richards J. Neurobiological Correlates of Social Conformity and Independence During Mental Rotation. Biol Psychiatry. 2005; 58(3): 245-53.

19. Ratcliff R, McKoon G. The diffusion decision model: theory and data for two-choice decision tasks. Neural Comput. 2008; 20(4): 873-922. 
20. Chen F, Krajbich I. Biased sequential sampling underlies the effects of time pressure and delay in social decision making. Nature Communications. 2018; 9(1): 35-7.

21. Kiani R, Shadlen MN. Representation of confidence associated with a decision by neurons in the parietal cortex. Science. 2009; 324(5928): 759-64.

22. Kiani R, Corthell L, Shadlen MN. Choice certainty is informed by both evidence and decision time. Neuron. 2014; 84(6): 1329-42.

23. Joo SJ, Katz LN, Huk AC. Decision-related perturbations of decision-irrelevant eye movements. Proc Natl Acad Sci U S A. 2016; 113(7): 1925-30.
24. O’Connell RG, Shadlen MN, Wong-Lin K, Kelly SP. Bridging neural and computational viewpoints on perceptual decision-making. Trends Neurosci. 2018; 41(11): 838-52.

25. Wang X-J. Probabilistic decision making by slow reverberation in cortical circuits. Neuron. 2002; 36(5): 955-68.

26. Wong KF. A recurrent network mechanism of time integration in perceptual decisions. Journal of Neuroscience. 2006; 26(4): 1314-28.

27. Voss A, Rothermund K, Brandtsta J. Interpreting ambiguous stimuli: Separating perceptual and judgmental biases. Journal of Experimental Social Psychology. 2008; 44(4): 1048-56. 